\title{
NEUROCYSTICERCOSIS PRESENTING WITH PAROXYSMAL SENSORY DISTURBANCE
}

\author{
Adriana Octaviana Dulamea \\ Department of Neurology, Fundeni Clinical Institute, \\ „Carol Davila“ University of Medicine and Pharmacy, Bucharest
}

\begin{abstract}
Author reports the case of a 28 years-old woman presenting episodes of paresthesia of the right arm with duration of approximately 4 days which remitted spontaneously, followed after two years by episodes of right hemiparesthesia. Brain MRI followed by brain stereotactic biopsy established the diagnosis of neurocysticercosis. Since electroencephalography showed no spike-wave abnormalities it was difficult to establish if symptoms were secondary to parietal lesion itself or were focal sensory seizures. After treatment with albendazole, methylprednisolone and sodium valproate the patient had a good recovery.
\end{abstract}

Keywords: neurocysticercosis, magnetic resonance imaging, brain stereotactic biopsy

\section{INTRODUCTION}

Neurocysticercosis is the most common helminthic infection affecting the central nervous system of humans being caused by Taenia solium. Two hosts are involved in the life cycle of this cestode, humans and pigs. Humans are definitive hosts for the adult tapeworm, whereas both pigs and humans may be intermediate hosts for the larval form (cysticercus). In the usual cycle of transmission, a few gravid proglottids from the adult tapeworm - attached to the intestinal wall of humans - are passed every few days with feces. Each proglottid liberates thousands of infective eggs to the environment. (1) Diversity of clinical manifestations of neurocysticercosis is related to differences in the number and location of the lesions within the nervous system and to the intensity of the host's immune response against the parasites. Seizures are the most common clinical form of presentation of neurocysticercosis, occurring in up to $80 \%$ of symptomatic infections. (2) Focal neurological signs have been described in $5 \%$ to $15 \%$ of patients (3) and often follow a chronic course resembling that of a brain tumor or of a focal granulomatous process (ie tuberculoma). (4) There were reports of cases of ischemic strokes (5), as well as intracranial hypertension related to hydrocephalus due to arachnoiditis, ventricular cysts, granular ependymitis or encephalitis. (6) Some patient with neurocysticercosis present cognitive decline and even dementia. (7) Intrasellar cysticerci (8), spinal arachnoiditis (9) and intraocular cysticerci (10) were also reported. Diagnosis is based on neuroimaging, positive serum immunoblot for the detection of anticysticercal antibodies and histologic demonstration of the parasite from brain biopsy. (1) On MRI vesicular (viable) cysticerci appear as small and rounded cysts that are well delineated from the brain parenchyma. Usually there is no edema or abnormal enhancement. A sizable proportion of those cysts have in their interior an eccentric hyperdense nodule representing the scolex, giving them "hole-with-dot" appearance, which is pathognomonic of neurocysticercosis. (11) Treatment comprises albendazole $15 \mathrm{mg} / \mathrm{kg} / \mathrm{d}$ from 3 days to 7 days depending on the type of cysts or praziquantel $30 \mathrm{mg} / \mathrm{kg}$ for 1 day or 
15 days, antiepileptic drug therapy may be added upon apparition of seizures as well as corticosteroids that are mandatory in heavy infection. (1)

\section{CASE REPORT}

Author reports the case of a 28 years-old woman without significant family or medical history which presented, after the birth of his child, episodes of paresthesia of the right arm with duration of approximately 4 days which remitted spontaneously, followed after two years by episodes of right hemiparesthesia. Neurologic examination showed: normal consciousness, no meningeal signs, hypoesthesia of the right side of the body, no pyramidal syndrome. General examination was normal. Ophtalmologic examination was normal. Brain MRI revealed two cystic like lesions, with thin wall that showed intense contrast enhancement, localized at the right lenticular level $(10 / 8 / 7 \mathrm{~mm})$ and left parietal level $(25 / 20 / 23 \mathrm{~mm})$; the parietal lesion is surrounded by important edema and inside shows a
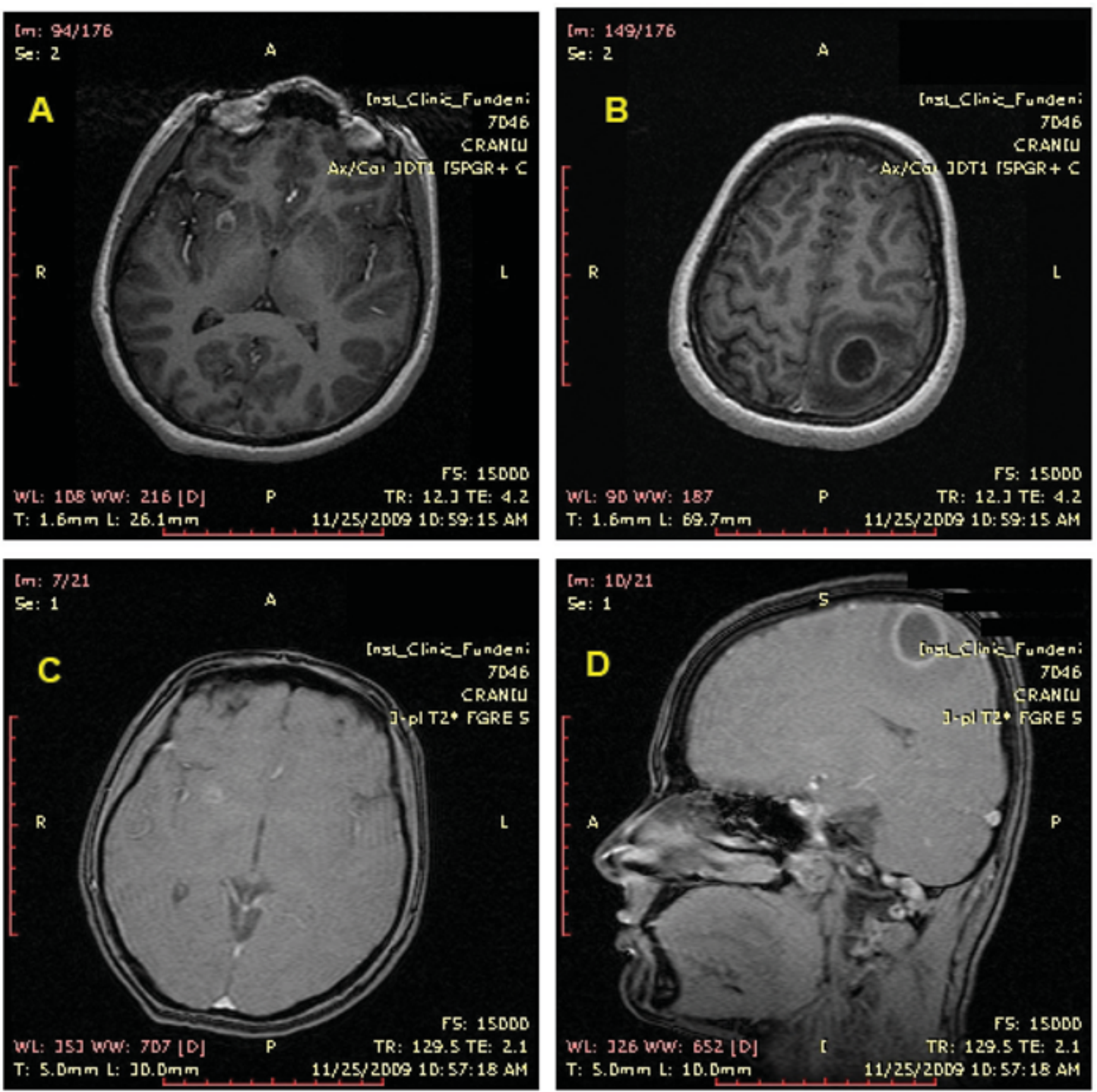

FIGURE 1. Brain MRI (A-B) T1 sequences with contrast enhancement, (C-D) T2 sequences: two cystic-like lesions, with thin wall that showed intense contrast enhancement, localized at the right lenticular level (10/8/7 mm) and left parietal level (25/20/23 $\mathrm{mm}$ ); the parietal lesion is surrounded by important edema and inside shows a small bud attached to the wall that appear hyperintense on T1 with contrast enhancement representing the scolex (,hole-withdot"sing) 
small bud attached to the wall that appear hyperintense on T1 with contrast enhancement representing the scolex ("hole-with-dot" sign); on diffusion weighted images the parietal lesion showed hyposignal except for a small bud attached to the wall that presented hypersignal. (Figure 1)

Electroencephalography showed no spike-wave abnormalities. The stereotactic brain biopsy and subsequent histology established the diagnosis of neurocysticercosis. Chest radiography and abdominal ultrasound were normal. Since electroencephalography showed no spike-wave abnormalities it was difficult to establish if symptoms were secondary to parietal lesion itself or were focal sensory seizures. The patient was treated with albendazole $15 \mathrm{mg} / \mathrm{kg} /$ day for 1 week associated to sodium valproate $600 \mathrm{mg} /$ day for 2 month and methylprednisolone $32 \mathrm{mg} /$ day for 2 weeks with progressive tapering of the dose. The patient experienced complete recovery.

Acknowledgements: Author thanks dr. Ioana Lupescu for selection and interpretation of brain magnetic resonance imaging.

\section{REFERENCES}

1. Del Brutto O.H. Neurocysticercosis. Neurohospitalist. 2014 Oct; 4(4):205-12. doi: 10.1177/1941874414533351.

2. Ndimubanzi P.C., Carabin H., Budke C.M., et al. A systematic review of the frequency of neurocysticercosis with a focus on people with epilepsy. PLoS Negl Trop Dis. 2010; 4(11):e870.

3. Carabin H., Ndimubanzi P.C., Budke C.M., et al. Clinical manifestations associated with neurocysticercosis: a systematic review. PLOS Negl Trop Dis. 2011; 5(5):e1152.

4. Fleury A., Carrillo-Mezo R., Flisser A., Sciutto E., Corona T. Subarachnoid basal neurocysticercosis: a focus on the most severe form of the disease. Expert Rev Anti-Infect Ther. 2011; 9(1): 123-133.

5. Marquez J.M., Arauz A. Cerebrovascular complications of neurocysticercosis. Neurologist. 2012; 18(1):17-22.

6. Garcia H.H., Del Brutto O.H. Neurocysticercosis: updated concepts about an old disease. Lancet Neurol. 2005; 4(10):653-661.

7. Forlenza O., Vieira A.H., Nobrea J.P.S., et al. Psychiatric manifestations in neurocysticercosis: a study of 38 patients from a neurologic clinics in Brazil. J Neurol Neurosurg Psychiatry. 1997; 62(6):612-616.

8. Del Brutto O.H., Del Brutto V.J. Intrasellar cysticercosis: a systematic review. Acta Neurol Belg. 2013; 113(3):225-227.

9. Chhiber S.S., Singh B., Bansal P., Pandita K.K., Razdan S., Singh J. Intramedullary spinal cysticercosis cured with medical therapy: case report and review of the literature. Surg Neurol. 2009; 72(6):765-769.

10. Madiubba S., Vishwanath K., Reddy G., Vemuganti G.K. Changing trends in ocular cysticercosis over two decades: an analysis of 118 surgically excised cysts. Indian J Med Microbiol. 2007; 25(3):214-219.

11. Garcia H.H., Del Brutto O.H., Nash T.E., et al. New concepts in the diagnosis and management of neurocysticercosis (Taenia solium). Am J Trop Med Hyg. 2005;72(1):3-9. 